\title{
Inheritance of Resistance to Leptosphaeria maculans in Brassica juncea
}

\author{
M. Keri, C. G. J. van den Berg, P. B. E. McVetty, and S. R. Rimmer
}

Department of Plant Science, University of Manitoba, Winnipeg, Manitoba, Canada R3T 2N2.

Accepted for publication 28 February 1997.

\begin{abstract}
Keri, M., van den Berg, C. G. J., McVetty, P. B. E., and Rimmer, S. R. 1997. Inheritance of resistance to Leptosphaeria maculans in Brassica juncea. Phytopathology 87:594-598.

The inheritance of resistance to Leptosphaeria maculans, the causal agent of black leg of crucifers, was studied in Brassica juncea. Three resistant accessions (UM3021, UM3043, and UM3323) and one susceptible accession (UM3132) of B. juncea were crossed in a complete diallel. Parents, $F_{1}$, and $F_{2}$ progenies were evaluated for all crosses using both cotyledon and stem inoculation. Cotyledon reaction was evaluated with two isolates of $L$. maculans, but stem reaction was evaluated with one isolate. Disease reactions observed for individual plants were the same for both inoculation methods and for both isolates of the pathogen for cotyledon reaction. No segregation was observed for the crosses between resistant accessions (UM3043 × UM3323 and UM3021 × UM3323),

but a few susceptible plants were observed in the $\mathrm{F}_{2}$ progeny of crosses between resistant parents (UM3021 $\times$ UM3043). This was probably due to heterozygosity in some parental plants of UM3021. For crosses between the susceptible parent and resistant parents, $\mathrm{F}_{1}$ plants for two crosses were all resistant. For cross UM3132 $\times$ UM3021, some susceptible plants occurred, which was also suggestive of heterozygosity in UM3021. Although resistance in $F_{1}$ was dominant, for $F_{2}$ populations, segregation fit either 13:3, 3:1, or 1:3 ratios, indicating that resistance can be either a dominant or recessive trait. $\mathrm{F}_{3}$ families derived from some susceptible $\mathrm{F}_{2}$ plants from crosses UM3021 × UM3132 and UM3043 × UM3132 were evaluated using the cotyledon inoculation method only. Segregation of $F_{2}$ plants and $\mathrm{F}_{3}$ families in crosses involving resistant and susceptible parents indicated that the resistance to L. maculans in B. juncea is controlled by two nuclear genes with dominant recessive epistatic gene action.
\end{abstract}

Black leg (stem canker), caused by Leptosphaeria maculans (Desmaz.) Ces. \& de Not., is an important disease in many Brassica species and may cause serious seed yield losses in oilseed brassica crops in many parts of the world $(6,10)$. The disease has been controlled primarily through the use of resistant cultivars. The genetics of resistance to L. maculans in Brassica spp. has been reviewed recently (11), but despite the importance of the disease, information on the number of genes involved in resistance and their effects and allelic relationships is rather limited. More knowledge concerning the genetics of resistance would be valuable for effective breeding for resistance to black leg in oilseed brassicas.

$B$. juncea (L.) Czern. (oilseed mustard) is more resistant to $L$. maculans at the seedling and adult plant stages than are $B$. rapa (turnip oilseed rape) and B. napus (oilseed rape), and this resistance is thought to be controlled by genes located on the bgenome $(12,13)$. There is considerable interest in western Canada to develop canola-quality $B$. juncea, because of its resistance to black leg and its superior drought tolerance. Resistance in $B$. juncea has been transferred to $B$. napus through interspecific hybridization (12). Since no information on the inheritance of resistance in $B$. juncea is available, this study was undertaken to determine the genetic control of resistance to L. maculans in this species.

\section{MATERIALS AND METHODS}

Plant material. In preliminary experiments, 296 accessions of B. juncea (University of Manitoba collection, obtained primarily from P. F. Knowles, University of California, Davis, and R. K. Downey, Agriculture and Agri-food Canada, Saskatoon) were

Corresponding author: S. R. Rimmer; E-mail address: rimmer@cc.umanitoba.ca

Publication no. P-1997-0411-01R

(c) 1997 The American Phytopathological Society evaluated for resistance to L. maculans (4), and four accessions (three resistant and one susceptible) were chosen to study the inheritance of resistance (Table 1). Single plants selected from the chosen accessions were inbred to the second generation selfed $\left(\mathrm{S}_{2}\right)$ level and crossed in a complete diallel. All plants that were used in crosses also were selfed to obtain third generation selfed $\left(\mathrm{S}_{3}\right)$ progeny. Racemes used for selfing or crossing were isolated in glycine bags to avoid pollen contamination. $F_{1}$ and $F_{2}$ populations were generated for all crosses, and $\mathrm{F}_{3}$ families derived from randomly selected susceptible $F_{2}$ plants were generated for the crosses UM3021 $\times$ UM3132 and UM3132 $\times$ UM3043. To facilitate interpretation of the data presented in Tables 2, 3, 4, and 5, crosses between the susceptible parent UM3132 and the resistant parents were designated as follows: UM3323 $\times$ UM3132 as cross I and the reciprocal as cross IR; UM3132 $\times$ UM3043 as cross IIR; and $\mathrm{UM} 3021 \times \mathrm{UM} 3132$ as cross III and the reciprocal as cross IIIR.

To evaluate the plants for disease reaction, seeds were planted separately in 5-cm jiffy pots (Koral flats; Koral Co., Bramalea, Ontario, Canada) filled with a soilless potting mixture (Metromix; W. R. Grace \& Co. Canada Ltd., Ajax, Ontario, Canada) composed of the following as basic ingredients: vermiculite, perlite, sphagnum peat moss, and nutrients (NPK and trace elements). All plants were maintained in a growth chamber at $24 / 20^{\circ} \mathrm{C}$ day/night temperatures with a 16-h photoperiod. After 3 weeks, seedlings were transplanted into 20 -cm fiber pots containing the soilless mixture. Plants were kept in a greenhouse with normal daylight, supplemented to $16 \mathrm{~h}$ with fluorescent light. Plants were fertilized regularly with a water-soluble formulation (20-20-20 NPK) containing micronutrients. Approximately $50 \mathrm{~S}_{2}$ plants and $200 \mathrm{~S}_{3}$ plants were evaluated for each accession, as well as $50 \mathrm{~F}_{1}$ and several hundred $F_{2}$ plants of each cross. Six and $18 \mathrm{~F}_{2}$-derived $F_{3}$ families were evaluated for the crosses between UM3132 $\times$ UM3043 (cross IIR) and between UM3021 × UM3132 (cross IIIR), respectively.

Pathogen isolates. Two isolates of L. maculans (P185-9 and Pl86-14), obtained from widely separated regions of Manitoba, 
Canada, were used in this study. Both isolates belong to pathogenicity group 2 , the predominant aggressive pathotype occurring in western Canada (7). To maintain pathogenicity, susceptible host plants were inoculated with the isolates using the cotyledon inoculation method (17). The infected cotyledons were surface-sterilized in 5\% sodium hypochlorite for 2 to $3 \mathrm{~min}$, rinsed twice in sterile distilled water, and transferred onto V8 juice agar plates
(800 $\mathrm{ml}$ of distilled water, $200 \mathrm{ml}$ of $\mathrm{V} 8$ juice, $0.7 \mathrm{~g}$ of calcium carbonate, and $15 \mathrm{~g}$ of agar) containing streptomycin sulfate (100 $\mathrm{mg} \mathrm{liter}^{-1}$ ) and rose bengal (40 mg liter ${ }^{-1}$ ). Plates were incubated under near-UV light or cool fluorescent light at room temperature to ensure high pycnidial production. After 5 to 7 days, plates were flooded with 5 to $10 \mathrm{ml}$ of sterile distilled water and gently scraped with a sterile glass slide to release the pycnidiospores.

TABLE 1. Description of Brassica juncea accessions and interaction phenotype on second $\left(\mathrm{S}_{2}\right)$ and third $\left(\mathrm{S}_{3}\right)$ generation selfs following cotyledon inoculation with isolate P186-14 of Leptosphaeria maculans

\begin{tabular}{|c|c|c|c|c|c|}
\hline \multirow[b]{2}{*}{ Accession } & \multirow[b]{2}{*}{ Country of origin } & \multirow[b]{2}{*}{ Name } & \multicolumn{2}{|c|}{ Cotyledon rating $^{z}$} & \multirow[b]{2}{*}{ General host reaction } \\
\hline & & & $\mathrm{S}_{2}$ & $\mathrm{~S}_{3}$ & \\
\hline UM3021 & Pakistan & $\ldots$ & $0.45 \pm 0.14$ & $0.25 \pm 0.06$ & Resistant \\
\hline UM3043 & Pakistan & $\ldots$ & $0.63 \pm 0.14$ & $0.25 \pm 0.05$ & Resistant \\
\hline UM3323 & China & $\mathrm{Ku}$ & $0.61 \pm 0.11$ & $0.30 \pm 0.05$ & Resistant \\
\hline UM3132 & Bangladesh & Rai-5 & $8.48 \pm 0.18$ & $8.72 \pm 0.07$ & Susceptible \\
\hline
\end{tabular}

${ }^{\mathrm{z}}$ Mean rating and standard deviation of interaction phenotype (0 to 9 scale) based on the cotyledon inoculation method (16).

TABLE 2. Observed segregation for host reaction, expected segregation ratios, chi-square values $\left(\chi^{2}\right)$, and probabilities $(P)$ for goodness of fit for $\mathrm{F}_{2}$ plants from crosses between accessions of Brassica juncea following cotyledon inoculation with isolate P186-14 of Leptosphaeria maculans

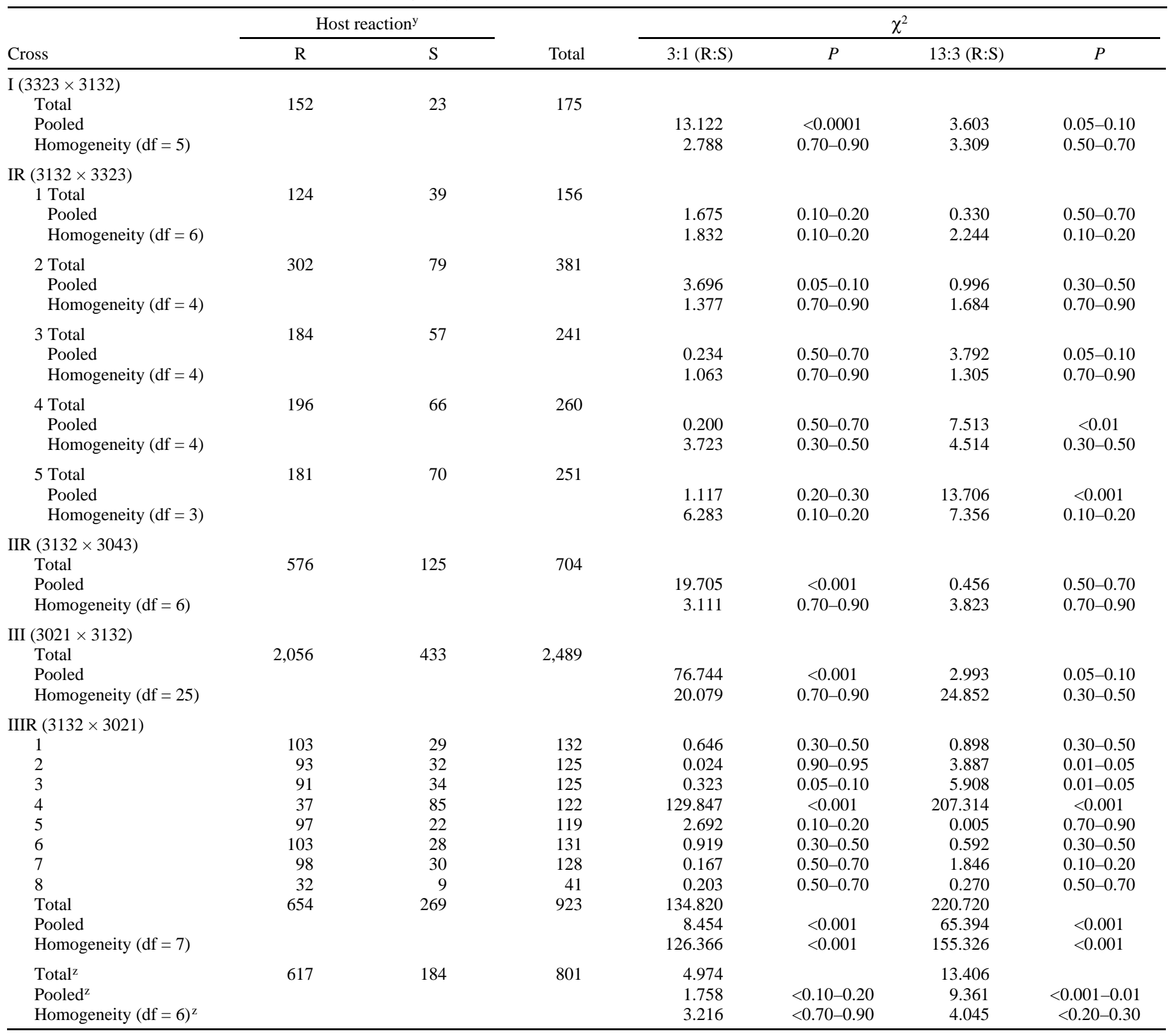

${ }^{y}$ Number of resistant (R) and susceptible (S) plants.

${ }^{\mathrm{z}}$ Cross IIIR 4 was excluded in pooling the data. 
The spore suspension was filtered through four layers of sterilized cheesecloth and centrifuged for $30 \mathrm{~min}$ at 3,000 $\times \mathrm{g}$. The supernatant was decanted, and the pelleted spores were suspended in 5 $\mathrm{ml}$ of sterile distilled water and stored at $-15^{\circ} \mathrm{C}$. For inoculation, the spore suspensions were thawed and the inoculum concentration was adjusted to $10^{7}$ spores $\mathrm{ml}^{-1}$. Using this method, the concentrated inoculum may be held frozen for 3 to 6 months without any noticeable loss in virulence of $L$. maculans.

Inoculation techniques. Cotyledon inoculation. Six days after seeding, cotyledons were wounded with a sterile needle and a 10$\mu \mathrm{l}$ droplet of the pycnidiospore suspension was placed onto the wound. The two isolates, Pl85-9 and Pl86-14, were each applied to one cotyledon of each plant. Inoculated plants were allowed to dry overnight before transfer to a growth chamber. Ten days after inoculation, the disease reactions on the cotyledons (cotyledon interaction phenotype [IP]) were rated using a 0 to 9 scale (17). $B$. napus cv. Westar was included in all inoculation tests as a susceptible check.

Stem inoculation. Plants were inoculated at the early bolting growth stage (GS 3.0 to 3.2 [3]) with isolate Pl86-14. A 10- $\mu$ l droplet of the pycnidiospore suspension was injected into the stem of each plant (between the second and third node) with a hypodermic syringe (model 5602; Beckton Dickinson, Rutherford, NJ). Lesion length and amount of girdling were rated 38 days after inoculation. Lesion length was rated using a 0 to 5 scale in which $0=$ no infection; $1=$ lesion length $<10 \mathrm{~mm} ; 2=$ lesion length 10 to $19 \mathrm{~mm} ; 3=$ lesion length 20 to $29 \mathrm{~mm} ; 4=$ lesion length $>30$ $\mathrm{mm}$; and $5=$ plant dead. Girdling was rated using a 0 to 5 scale as previously reported $(9,16)$. Stem disease reaction (adult IP) was calculated as the sum of the ratings for girdling and lesion length. IPs accompanied by sporulation of the fungus on the host tissue were considered indicative of susceptibility. B. napus cv. Westar was included in all inoculation tests as a susceptible check.

Statistical analysis. The chi-square test was used to test the goodness of fit of segregation ratios for all data (15). The validity of pooling $\mathrm{F}_{2}$ populations from the same cross was tested with the chi-square test for homogeneity.

\section{RESULTS}

Parents. Similar cotyledon IPs were observed with both isolates (P185-9 and P186-14) of L. maculans. Consequently, only data for isolate Pl86-14 are presented. The mean IP of the three resistant accessions was less than 0.7 in the $S_{2}$ and $S_{3}$ generations (Table 1), and the mean cotyledon IP for the susceptible parent was greater than 8.4 in both the $S_{2}$ and $S_{3}$ generations. The cotyledon IP for the resistant accessions ranged from 0 to 3 and, for the susceptible accession, from 5 to 9 . Following stem inoculation, the adult IP was less than 0.6 for resistant accessions and greater than 7.0 for the susceptible accessions. At the adult stage, IP ranged from 0 to 3 for resistant accessions and from 6 to 8 for the susceptible accession. No segregation was observed in the $S_{2}$ or $S_{3}$

TABLE 3. Interaction phenotype (IP), expected segregation ratio, chi-square values $\left(\chi^{2}\right)$, and probabilities $(P)$ for goodness of fit for $\mathrm{F}_{3}$ families derived from susceptible $F_{2}$ plants of the cross UM3132 $\times$ UM3043 (cross II) following cotyledon inoculation with isolate P186-14 of Leptosphaeria maculans

\begin{tabular}{|c|c|c|c|c|c|c|}
\hline \multirow{2}{*}{$\begin{array}{l}\mathrm{F}_{3} \text { family } \\
\text { number }\end{array}$} & \multicolumn{2}{|c|}{ Host reaction $^{2}$} & \multirow[b]{2}{*}{ Total } & \multirow{2}{*}{$\begin{array}{l}\text { Expected ratio } \\
\text { (R:S) }\end{array}$} & \multirow[b]{2}{*}{$\chi^{2}$} & \multirow[b]{2}{*}{$P$} \\
\hline & $\mathrm{R}$ & 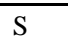 & & & & \\
\hline $1-1$ & 1 & 47 & 48 & $0: 1$ & & \\
\hline $1-2$ & 1 & 46 & 47 & $0: 1$ & & \\
\hline $1-3$ & 16 & 31 & 47 & $1: 3$ & 2.050 & $0.10-0.25$ \\
\hline $6-1$ & 11 & 36 & 47 & $1: 3$ & 0.064 & $0.75-0.90$ \\
\hline $7-1$ & 16 & 32 & 48 & $1: 3$ & 1.778 & $0.10-0.25$ \\
\hline $7-2$ & 1 & 44 & 45 & $0: 1$ & & \\
\hline
\end{tabular}

${ }^{\mathrm{z}}$ Number of resistant (R) and susceptible (S) plants. progeny of any accession. Classification of cotyledon IP or adult IP of individual plants for subsequent $F_{1}, F_{2}$, and $F_{3}$ generations followed the same classifications as for the parental material. One exception to this is explicitly described below.

F $_{1}$ progeny. For crosses involving UM3323 $\times$ UM3132 (crosses I and IR) and UM3132 $\times$ UM3043 (cross IIR), the $F_{1}$ progeny were uniformly resistant with a mean cotyledon IP less than 0.43 . Segregation for resistance and susceptibility was observed in the $\mathrm{F}_{1}$ progeny for the cross UM3021 $\times$ UM3132 (cross III) (209 resistant:33 susceptible) and the reciprocal (cross IIIR) (449 resistant:41 susceptible), indicating heterozygosity in some parental plants of UM3021.

Following stem inoculation, adult IP ranged from 0 to 1 in the crosses between resistant accessions. For the crosses UM3323 $\times$ UM3132 (crosses I and IR) and UM3132 × UM3043 (cross IIR), adult IP ranged from 0 to 1 . For the cross UM3021 $\times$ UM3132 (crosses III and IIIR), adult IP ranged from 0 to 5; most IPs were 0 to 1 , as in the other crosses, and were classified as resistant. However, some plants were of adult IP 4 or 5 . These were characterized by purple streaking that originated from the point of inoculation of stems; fungal sporulation occurred on stems of such plants 4 weeks after inoculation. Consequently, these plants, which also had a cotyledon IP of 5, were classified as susceptible.

$\mathbf{F}_{2}$ progeny. With the cotyledon inoculation, $\mathrm{F}_{2}$ progeny for the crosses between susceptible and resistant accessions fit both a 13:3 and 3:1 resistant/susceptible segregation ratio. Most $F_{2}$ progeny for the crosses of UM3132 with the resistant accessions were best fit to a 13 resistant:3 susceptible segregation ratio (Table 2). However, some progeny in the reciprocal crosses of UM3323 $\times$ UM3132 (cross IR) and UM3021 × UM3132 (cross IIIR) fit only a 3 resistant:1 susceptible segregation ratio. Chi-square tests for homogeneity on all families from the reciprocal cross of UM3021 $\times$ UM3132 (cross IIIR) were significant for both 13:3 $(P<0.001)$ and 3:1 $(P<0.001)$ resistant/susceptible segregation ratios. One family (cross IIIR 4 , Table 2 ), which best fit a 1 resistant:3 susceptible segregation ratio (chi-square $=1.847$ ), was then excluded in the tests for homogeneity; the pooled data for this cross was then homogeneous for a 3 resistant: 1 susceptible segregation ratio. The chi-square test for homogeneity of the pooled $\mathrm{F}_{2}$ data for the other crosses of UM3132 with resistant accessions was not significant for a 13:3 ratio of resistant/susceptible plants (Table 2). Thus, resistance in the B. juncea UM3323, UM3043, and UM3021

TABLE 4. Interaction phenotype (IP), expected segregation ratio, chi-square values $\left(\chi^{2}\right)$, and probabilities $(P)$ for goodness of fit for $\mathrm{F}_{3}$ families derived from susceptible $F_{2}$ plants of the cross UM3132 $\times$ UM3021 (cross IIIR) following cotyledon inoculation with isolate P186-14 of Leptosphaeria maculans

\begin{tabular}{|c|c|c|c|c|c|c|}
\hline \multicolumn{3}{|c|}{$\mathrm{F}_{2}$ family Interaction phenotype ${ }^{\mathrm{z}}$} & \multirow[b]{2}{*}{ Total } & \multirow{2}{*}{$\begin{array}{l}\text { Expected } \\
\text { ratio }(\mathrm{R}: \mathrm{S})\end{array}$} & \multirow[b]{2}{*}{$\chi^{2}$} & \multirow[b]{2}{*}{$P$} \\
\hline number & $\mathrm{R}$ & $\mathrm{S}$ & & & & \\
\hline $3-1$ & 11 & 41 & 52 & $1: 3$ & 0.231 & $0.50-0.75$ \\
\hline $3-2$ & 10 & 35 & 45 & $1: 3$ & 0.067 & $0.75-0.90$ \\
\hline $3-3$ & 0 & 44 & 44 & $0: 1$ & & \\
\hline $3-4$ & 29 & 15 & 44 & $3: 1$ & 1.939 & $0.10-0.25$ \\
\hline $3-5$ & 21 & 23 & 44 & $1: 3$ & 2.735 & $0.05-0.10$ \\
\hline $3-6$ & 11 & 34 & 45 & $1: 3$ & 0.007 & $0.90-0.95$ \\
\hline $4-1$ & 1 & 44 & 45 & $0: 1$ & & \\
\hline $4-2$ & 11 & 31 & 42 & $1: 3$ & 0.000 & $>0.95$ \\
\hline $4-3$ & 16 & 32 & 48 & $1: 3$ & 1.361 & $0.25-0.50$ \\
\hline $4-4$ & 14 & 26 & 40 & $1: 3$ & 1.633 & $0.20-0.50$ \\
\hline $4-5$ & 13 & 33 & 46 & $1: 3$ & 0.116 & $0.75-0.90$ \\
\hline $4-6$ & 10 & 32 & 42 & $1: 3$ & 0.000 & $>0.95$ \\
\hline $8-1$ & 0 & 47 & 47 & $0: 1$ & & \\
\hline $8-2$ & 30 & 16 & 46 & $3: 1$ & 2.348 & $0.10-0.25$ \\
\hline $8-3$ & 12 & 34 & 46 & $1: 3$ & 0.000 & $>0.95$ \\
\hline $8-4$ & 11 & 29 & 40 & $1: 3$ & 0.033 & $0.75-0.90$ \\
\hline $8-5$ & 16 & 31 & 47 & $1: 3$ & 1.596 & $0.25-0.50$ \\
\hline $8-6$ & 0 & 53 & 53 & $0: 1$ & & \\
\hline
\end{tabular}

${ }^{\mathrm{z}}$ Number of resistant (R) and susceptible (S) plants. 
lines was controlled by two genes with dominant recessive epistatic interaction. A consequence of this model is that some susceptible $\mathrm{F}_{2}$ genotypes, when selfed, will segregate for resistance in $\mathrm{F}_{3}$ generation. A brief outline of this genetic model is presented at the beginning of the Discussion.

Following cotyledon inoculation, all plants in the $\mathrm{F}_{2}$ populations of the crosses between the resistant parents, UM3323 $\times$ UM3043 and UM3323 $\times$ UM3021, and their reciprocals were resistant (data not shown). This indicates that the resistance genes in UM3323 are allelic to those in UM3043 and UM3021. The $F_{2}$ populations from the cross UM3021 $\times$ UM3043 and their reciprocal were predominantly resistant (1,452 resistant:74 susceptible). Sixty-three plants with IP 5 and 11 plants with IP 7, distributed over several families, were observed in this cross and its reciprocal; these plants could be due to the heterogeneous and heterozygous genetic make-up of UM3021.

Adult IP ranged from 0 to 1 in the crosses between resistant accessions following stem inoculation. In the crosses of resistant with the susceptible parent (crosses I, II, and III) and their reciprocals, adult IP ranged from 0 to 9 . Plants with a rating 0 to 3 were classified as resistant, and plants with a rating from 4 to 9 were classified as susceptible. The classification for susceptibility was based on the fact that, with this range of adult IP, the pathogen was able to colonize and sporulate on the inoculated plants. The classification of individual plants as either susceptible or resistant, based on the IP ratings, was the same at the cotyledon and adult plant stages.

$\mathbf{F}_{\mathbf{3}}$ families. Although most $\mathrm{F}_{2}$ populations derived from crosses between resistant and susceptible lines best fit a 13 resistant: 3 susceptible segregation ratio, some populations in the reciprocal crosses of UM3323 $\times$ UM3132 (cross IR) and UM3021 × UM3132 (cross IIIR) best fit a 3 resistant: 1 susceptible segregation ratio, and one population (cross IIIR 4) in the reciprocal cross of UM3021 $\times$ UM3132 (cross IIIR) best fit a 1 resistant:3 susceptible segregation ratio. The hypothesis of two genes with dominant recessive epistatic interaction was then tested further by evaluating $\mathrm{F}_{3}$ families derived from susceptible $F_{2}$ plants from the populations of UM3021 × UM3132 (cross IIIR), which did not fit a 13 resistant: 3 susceptible ratio. In addition, susceptible plants derived from some $F_{2}$ populations from the cross UM3132 × UM3043 (cross
IIR) and cross UM3021 $\times$ UM3132 (cross IIIR), which did fit a 13 resistant: 3 susceptible ratio, were also evaluated in the $F_{3}$ generation. As predicted by the hypothesis, data from the $F_{3}$ families derived from susceptible $F_{2}$ plants of the crosses UM3132 $\times$ UM3043 (cross IIR) and UM3021 × UM3132 (cross IIIR) fit a 1:3 or $0: 1$ segregation ratio of resistant/susceptible plants (Tables 3 and 4 , respectively). However, two $\mathrm{F}_{3}$ families of the 24 tested fit a 3 resistant: 1 susceptible ratio, a ratio not explainable by the dominant recessive epistatic hypothesis (Table 4). Overall, these results confirm that resistance to $L$. maculans in $B$. juncea lines UM3323, UM3043, and UM3021 is controlled by two nuclear genes with dominant recessive epistatic interaction.

\section{DISCUSSION}

Results from this study indicate that two gene pairs interact to determine both seedling and stem resistance to L. maculans in $B$. juncea. With dominant recessive epistasis, one gene (A/a) confers resistance in a dominant manner (i.e., $\mathrm{A} / \mathrm{A}$ or $\mathrm{A} / \mathrm{a}$ ) and a second gene $(B / b)$ in a recessive manner (i.e., b/b). Presence of the Aallele at the first locus or the b-allele in homozygous state on the second locus is required for the resistant IP. A 3:1, 1:3, or both resistant/susceptible segregation ratios were observed for some $F_{2}$ populations from resistant $\times$ susceptible crosses. This suggests that the resistant accessions were segregating for the first locus (A/a) only. Resistant genotypes heterozygous for at least one locus are $\mathrm{AaBB}, \mathrm{Aabb}, \mathrm{AaBb}$, and the heterozygous susceptible genotype is aaBb. Only the genotypes $\mathrm{AaBB}$ and $\mathrm{aaBb}$, upon selfing, will result in a 3:1 and 1:3 resistant/susceptible segregation ratio, respectively. The genotypes $\mathrm{AaBB}$ and $\mathrm{aaBb}$ may be obtained if the genotypes of the resistant parents used in crosses with the homozygous susceptible genotype (aaBB) are AABb and Aabb, respectively. Thus, the presence of families with $3: 1,1: 3$, or both resistant/susceptible segregation ratios indicate that the resistant accessions (UM3021, UM3043, and UM3323) used in the study were segregating for at least one locus.

That the two genes interact in a dominant recessive manner is supported by the segregation for resistance in $\mathrm{F}_{3}$ families originating from single susceptible $F_{2}$ plants. Overall (22 of 24 families), $\mathrm{F}_{3}$ families fit a $0: 1$ or $1: 3$ resistant/susceptible segregation

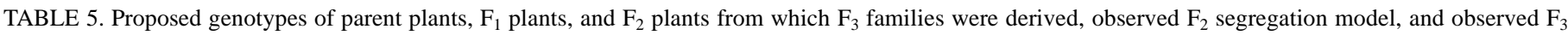

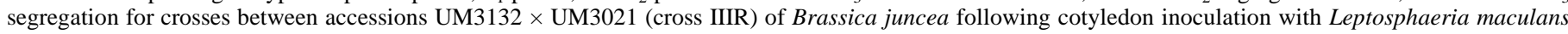
isolate P186-14

\begin{tabular}{|c|c|c|c|c|c|c|}
\hline $\begin{array}{l}\text { Cross IIIR } \\
\text { families }\end{array}$ & $\begin{array}{l}\text { Proposed genotype of } \\
\text { resistant parent plants }\end{array}$ & $\begin{array}{l}\text { Expected } \mathrm{F}_{1} \\
\text { genotypes }\end{array}$ & $\begin{array}{c}\text { Proposed genotype } \\
\text { of } F_{1} \text { plant }\end{array}$ & $\begin{array}{c}\text { Observed } \mathrm{F}_{2} \text { ratio } \\
(\mathrm{R}: \mathrm{S})\end{array}$ & $\begin{array}{l}\text { Proposed genotype of } \mathrm{F}_{2} \\
\text { plant to } \mathrm{F}_{3} \text { family }\end{array}$ & $\begin{array}{c}\text { Observed } \mathrm{F}_{3} \text { ratio } \\
(\mathrm{R}: \mathrm{S})\end{array}$ \\
\hline 1 & $\mathrm{AABb}$ & $\mathrm{AaBb} / \mathrm{AaBB}$ & $\begin{array}{l}\mathrm{AaBb} \\
\mathrm{AaBB}\end{array}$ & $\begin{array}{r}13: 3 \\
3: 1\end{array}$ & & \\
\hline 2 & $\mathrm{AaBB}$ or $\mathrm{AABb}$ & $\begin{array}{c}\mathrm{AaBB} / \mathrm{aaBB} \\
\text { (only } 1 \text { plant selected) }\end{array}$ & $\mathrm{AaBB}$ & $3: 1$ & & \\
\hline $3^{z}$ & $\mathrm{AABb}$ & $\mathrm{AaBb} / \mathrm{AaBB}$ & $\begin{array}{l}\mathrm{AaBb} \\
\mathrm{AaBB}\end{array}$ & $\begin{array}{r}13: 3 \\
3: 1\end{array}$ & $\begin{array}{l}\mathrm{aaBb} \\
\mathrm{aaBB} \\
\mathrm{AaBB}\end{array}$ & $\begin{array}{l}1: 3 \\
0: 1 \\
3: 1\end{array}$ \\
\hline $4^{z}$ & Aabb & $\mathrm{AaBb} / \mathrm{aaBb}$ & $\mathrm{aaBb}$ & $1: 3$ & $\begin{array}{l}\mathrm{aaBB} \\
\mathrm{aaBb}\end{array}$ & $\begin{array}{l}0: 1 \\
1: 3\end{array}$ \\
\hline 5 & $\mathrm{AABb}$ & $\mathrm{AaBB} / \mathrm{AaBb}$ & $\begin{array}{l}\mathrm{AaBb} \\
\mathrm{AaBB}\end{array}$ & $\begin{array}{r}13: 3 \\
3: 1\end{array}$ & & \\
\hline 6 & $\mathrm{AABb}$ & $\mathrm{AaBB} / \mathrm{AaBb}$ & $\begin{array}{l}\mathrm{AaBb} \\
\mathrm{AaBB}\end{array}$ & $\begin{array}{r}13: 3 \\
3: 1\end{array}$ & & \\
\hline 7 & $\mathrm{AABb}$ & $\mathrm{AaBB} / \mathrm{AaBb}$ & $\begin{array}{l}\mathrm{AaBb} \\
\mathrm{AaBB}\end{array}$ & $\begin{array}{r}13: 3 \\
3: 1\end{array}$ & & \\
\hline $8^{z}$ & & $\mathrm{AaBB} / \mathrm{AaBb}$ & $\begin{array}{l}\mathrm{AaBb} \\
\mathrm{AaBB}\end{array}$ & $\begin{array}{r}13: 3 \\
3: 1\end{array}$ & $\begin{array}{l}\mathrm{a} a \mathrm{BB} \\
\mathrm{aaBb} \\
\mathrm{AaBB}\end{array}$ & $\begin{array}{l}0: 1 \\
1: 3 \\
3: 1\end{array}$ \\
\hline
\end{tabular}

y Resistant parent plants (accession UM3021). Genotype of susceptible parent (UM3132) is assumed to be aaBB.

${ }^{\mathrm{z}} \mathrm{F}_{3}$ plants tested and used. 
ratio. The two families that fit a 3 resistant: 1 susceptible segregation ratio were unexpected and not predicted by the dominant recessive model. It is very unlikely that these observations could be due to experimental error, such as the misclassification of IP scored. However, if $\mathrm{F}_{2}$ plants with the genotype $\mathrm{AaBB}$ were scored as susceptible, a 3 resistant: 1 susceptible segregation ratio would be observed in their $F_{3}$ families. Proposed genotypes for parents, $F_{1}$, and $F_{2}$ progeny from which $F_{3}$ families were derived, and the obtained $F_{3}$ for UM3132 $\times$ UM3021 (cross IIIR) are presented in Table 5.

Using cotyledon inoculation, Mithen and Lewis (8) found that seedling resistance in B. alboglabra is controlled by dominant, epistatic genes (15 resistant:1 susceptible segregation ratio). Delwiche (2) found two linked, dominant genes in B. napus. Sawatsky (14) reported that seedling resistance was controlled by a single recessive gene in B. napus and, using stem inoculation, identified two dominant genes conferring stem resistance at the adult plant stage. In this study, we identified two epistatic genes in $B$. juncea that confer resistance at both the seedling and adult plant stages. To our knowledge, resistance inherited by dominant recessive epistasis is unusual. It has been reported for resistance in wheat to races of Puccinia striiformis (1). Virulence/avirulence in Melampsora lini, the flax rust pathogen, has also been reported to be controlled by dominant recessive interacting genes (5).

Previously, 296 B. juncea accessions were evaluated for disease reaction to $L$. maculans isolates. Most of the accessions tested were resistant to the L. maculans isolates used (4). UM3132, the susceptible parent used in this study, was one of only three accessions found to be susceptible to the two L. maculans isolates used (4). Thus, it appears that resistance to L. maculans is the norm in B. juncea. If this is the case and resistance is controlled by two genes with dominant recessive epistasis, then only certain genotypes of $B$. juncea are likely to be present for resistance to be homogeneous in the absence of selection pressure. Genotypes homozygous for at least one locus for resistance are AABB, AAbb, aabb, and the homozygous susceptible genotype is aaBB. Crosses between genotypes $\mathrm{AABB}$ and $\mathrm{AAbb}$ result only in resistant progeny (genotype $\mathrm{AABb}$ ). If the genotype aabb occurs among accessions of $B$. juncea, any crosses of this genotype with genotype $\mathrm{AABB}$ or $\mathrm{AAbb}$ will result in segregation for susceptibility and resistance only in crosses with genotype AABB. We chose three resistant accessions at random in this study, and segregation for susceptibility was not observed for UM3323 $\times$ UM3021 or UM3323 × UM3043. However, with UM3021 × UM3043, a small frequency of susceptible plants were observed (1,452 resistant:74 susceptible) in the $F_{2}$ populations with the susceptible plants occurring in a number of the families tested. The low number of susceptible plants occurring in this cross cannot simply be explained by the genotypes AABB, AAbb, or aabb above; we have no genetic explanation for this data, although it could be due to a variety of reasons including the heterogeneous and heterozygous genetic make-up of UM3021, other epistatic effects, or associative and dissociative effects of the genes.

The resistance of $B$. juncea to $L$. maculans is well known, and attempts have been made to transfer this resistance to $B$. napus $(12,13)$. The inheritance of resistance has not been studied in resistant lines derived from interspecific crosses. Results from this study allow the evaluation of the efficiency of gene transfer using interspecific hybridization. The dominant recessive epistasis model suggests that both dominant and recessive control of resistance may occur in the Brassica-L. maculans pathosystem. The elucidation of the genetic control of resistance may depend on, or may be determined by, the genotype of the original parent used in a genetic study, and on the number of $F_{1}$ plants of a cross that were selected and used to generate $F_{2}$ populations. In this study, up to eight $F_{1}$ plants were randomly selected and used to generate $F_{2}$ populations for crosses between resistant $\times$ susceptible accessions. With regard to transferring the resistance of $B$. juncea to $L$. maculans from $B$. juncea to the major brassicas (B. napus, B. oleracea, and $B$. rapa), this model suggests that if the $B$. juncea type of resistance is to be maintained, both genes may have to be transferred to susceptible cultivars, since the dominant gene has major effects on the expression of IP and the recessive gene has modifying effects on the expression of the dominant allele. However, it may be that the transfer of one of the two genes will result in expression of resistance in the recipient species as either a dominant or a recessive character. The possibility of the transfer of a single gene would be most desirable for plant breeding purposes.

\section{ACKNOWLEDGMENTS}

Research was supported by grants from Zeneca Seeds Canada and the IRAP program of the National Research Council of Canada.

\section{LITERATURE CITED}

1. Chen, X.-M., and Line, R. F. 1992. Identification of stripe rust resistance genes in wheat genotypes used to differentiate North American races of Puccinia striiformis. Phytopathology 82:1428-1434.

2. Delwiche, P. A. 1980. Genetic aspects of black leg (Leptosphaeria maculans) resistance in rapeseed (Brassica napus). Ph.D. thesis. University of Wisconsin, Madison, WI.

3. Harper, F. R., and Berkenkamp, B. 1975. Revised growth-stage key for Brassica campestris and B. napus. Can. J. Plant Sci. 55:657-658.

4. Keri, M. 1991. Resistance of Brassica juncea Czern. \& Coss to blackleg disease caused by Leptosphaeria maculans (Desm.) Ces. \& de Not. M.Sc. thesis. University of Manitoba, Winnipeg, Manitoba, Canada.

5. Lawrence, G. J., Mayo, G. M. E., and Shepherd, K. W. 1981. Interactions between genes controlling pathogenicity in the flax rust fungus. Phytopathology 71:12-19.

6. McGee, D. C., and Emmett, R. W. 1977. Blackleg (Leptosphaeria maculans (Desm.) Ces. et de Not.) of rapeseed in Victoria: Crop losses and factors which affect disease severity. Aust. J. Agric. Res. 28:47-51.

7. Mengistu, A., Rimmer, S. R., Koch, E., and Williams, P. H. 1991. Pathogenicity grouping of isolates of Leptosphaeria maculans on Brassica napus cultivars and their disease reaction profiles on rapid-cycling brassicas. Plant Dis. 75:1279-1282.

8. Mithen, R. F., and Lewis, B. G. 1988. Resistance to Leptosphaeria maculans in hybrids of Brassica oleracea and Brassica insularis. J. Phytopathol. 123:253-258.

9. Newman, P. L. 1984. Differential host-parasite interaction between oilseed rape and Leptosphaeria maculans, the causal fungus of stem canker. Plant Pathol. 33:205-210.

10. Newman, P. L., and Bailey, D. J. 1987. Screening for resistance to canker (Leptosphaeria maculans) in winter oilseed rape (Brassica napus spp. oleifera). Plant Pathol. 36:346-354.

11. Rimmer, S. R., and van den Berg, C. G. J. 1992. Resistance of oilseed Brassica spp. to blackleg caused by Leptosphaeria maculans. Can. J. Plant Pathol. 14:56-66.

12. Roy, N. N. 1984. Interspecific transfer of Brassica juncea-type blackleg resistance to Brassica napus. Euphytica 33:295-303.

13. Sacristán, M. D., and Gerdemann, M. 1986. Different behavior of Brassica juncea and Brassica carinata as sources of Phoma lingam resistance in experiments of interspecific transfer to B. napus. Z. Pflanzenzücht. 97:304-314.

14. Sawatsky, W. M. 1989. Evaluation of screening techniques for resistance to Leptosphaeria maculans and genetic studies of resistance to the disease in Brassica napus. M.Sc. thesis. University of Manitoba, Winnipeg, Manitoba, Canada.

15. Steel, R. G. D., and Torrie, J. H. 1980. Principles and Procedures of Statistics: A Biometrical Approach. McGraw-Hill Book Co., New York.

16. van den Berg, C. J. G., Rimmer, S. R., and Parks, P. 1993. Comparison of scales for rating severity of blackleg in canola. Can. J. Plant Pathol. 15:49-53.

17. Williams, P. H. 1985. Crucifer Genetics Cooperative (CrGC) Resource Book. 1985. Department of Plant Pathology, University of Wisconsin, Madison, WI. 